\title{
Differential- and common-mode radiofrequency interference filters based on complementary split-ring resonators: a conduction and radiation impact analysis
}

\begin{abstract}
This paper aims to analyse the design techniques of differential transmission lines loaded with metamaterials. Specifically, complementary split-ring resonators (CSRRs) were etched on the ground plane of a microstrip transmission line in order to mitigate both differential- and common-mode propagation in differential signalling. Several prototypes were manufactured and characterised in printed circuit boards (PCBs). The topologies under test were measured and compared as radiofrequency interference (RFI) filters, taking into account frequency response, signal integrity, and near- and far-field radiation impact with regard to a reference (solid ground) board.
\end{abstract}

Keywords: Metamaterials; complementary split-ring resonators; radiofrequency interference; differentialmode filter; common-mode filter. 


\section{INTRODUCTION}

In theory, differential signalling provides both high immunity to noise and crosstalk and a low level of radiated electromagnetic interference (EMI). In practice, small asymmetries caused by trace length, time skews or imbalances between the two transmitted signals lead to the conversion of some of the differentialmode (DM) signals into common-mode (CM) ones, which can critically affect the electromagnetic compatibility (EMC) performance of high data-rate transmission in high-speed digital electronic buses and circuits. Various compact common-mode filters based on metamaterials such as electromagnetic bandgaps (EBGs) [1] or negative-permittivity metamaterials [2] have been presented to overcome this issue.

Over the past decade, complementary split-ring resonators (CSRRs) [3] have been used as subwavelength negative effective media $(\varepsilon<0)$ resonators in order to design microwave devices and antennas due to their compact size, low cost and high quality factor (Q). CSRRs are metamaterials that satisfy the condition $\lambda<<p$ (where $\mathrm{p}$ is the period between particles and $\lambda$ the propagation signal wavelength) and are able to generate narrow stop-band responses that can be tuned to undesired propagation bands. The cost of manufacturing these resonators is negligible since they are implemented by means of conventional etching techniques, typically on the PCB ground plane. In particular, several studies have shown the excellent qualities of CSRRs for filtering and mitigating radiofrequency interference (RFI) and high-frequency EMC issues [4-7]. Recently, a new common-mode filter design based on CSRRs was presented for Ethernet LAN applications [8]. The use of differential- and common-mode filters based on these kinds of metamaterials has also been reported [9].

This paper analyses both differential- and common-mode filters based on CSRRs. It reports the electromagnetic simulation and experimental validation of the differential- and common-mode filters, as well as the signal integrity performance for the considered cases. Moreover, a near- and far-field radiation analysis is performed to investigate the impact in terms of radiated emission due to the alteration of the solid ground plane (as a result of the presence of the etched resonators). 
The remainder of the paper is organised as follows. Section 2 describes CSRR differential- and commonmode layout topologies and their S-parameter frequency response. Section 3 addresses the signal integrity issues of the different topologies under test. Section 4 discusses the simulated and experimental data from the near- and far-field radiation analysis. Finally, Section 5 presents the main conclusions.

\section{DIFFERENTIAL-AND COMMON-MODE RFI FILTERS BASED ON CSRRS}

Fig. 1 illustrates two coupled rectangular CSRRs etched on a ground plane and their main design geometric parameters. Essentially, CSRRs consist of a pair of rings etched on the ground plane with apertures on opposite sides that are mainly excited by means of an axial time-varying electric field. CSRRs behave as an LC tank that can be coupled to one or both microstrip host lines through the transmission line capacitance. Therefore, CSRR resonance frequency is given by (1), where $L_{C}$ and $C_{C}$ represent the equivalent inductance and capacitance of the resonator, respectively.

$$
\mathrm{f}_{\mathrm{CSRR}}=\frac{1}{2 \pi \sqrt{\mathrm{L}_{\mathrm{C}} \mathrm{C}_{\mathrm{C}}}}
$$

The design frequency operation was set at $2.4 \mathrm{GHz}$ due to its practical interest for applications located in the Industrial, Scientific and Medical (ISM) radio band. To design the CSRRs' dimensions, an initial procedure based on considering their dual counterpart (SRRs) was followed according to [10-11]. Due to the number of geometric parameters, a multiple tuning procedure was developed to determine the final dimensions required to achieve the necessary operating resonance frequency. The width and separation of the rings, as well as the inter-resonator distance, were set at the minimum resolution required for the manufacturing process, $c=d=e=0.3 \mathrm{~mm}$ (all PCBs were manufactured with an LPKF S62 drilling machine). All the rings were designed with the same dimensions in order to enhance the stop-band effect at 2.4 GHz to mitigate potential ISM band interference. The perimeter of the designed CSRRs had the following dimensions: $a=6.3 \mathrm{~mm}$ and $b=5.0 \mathrm{~mm}$. 
Fig. 2(a) shows the considered microstrip differential topology. All three PCBs considered had $50 \Omega$ microstrip access lines to ensure good matching performance in testing. The coupled transmission lines' dimensions were as follows: width $w=1.18 \mathrm{~mm}$, separation $s=2.1 \mathrm{~mm}$ and length $l=32 \mathrm{~mm}$, with a total circuit area of $65.2 \times 47.8 \mathrm{~mm}^{2}$. Note that $45^{\circ}$ access lines were added for convenience in order to insert the port connectors. All of the designed PCBs were implemented in the commercial Rogers RO3010 substrate (dielectric constant, $\varepsilon_{r}=10.2$; thickness, $h=1.27 \mathrm{~mm}$ ) with layers of copper (thickness, $t=35 \mu \mathrm{m}$ ). Fig. 2(b) shows the common- and differential-mode CSRR filter (CSRR-CM-DM). An array of 6-stage CSRRs was etched underneath each individual microstrip line. As demonstrated in [9], the equivalent electrical model of the CSRR-CM-DM topology for the differential (odd) and common (even) modes was of the same nature. In both cases, a parallel notch resonance arises and, therefore, a stop-band frequency response is expected. Consequently, both propagation modes are suppressed at the CSRRs' resonance frequency. Conversely, Fig. 2(c) shows the topology of the common-mode CSRR filter (CSRR-CM). In this case, a 6stage array of CSRRs was etched underneath both microstrip coupled lines. As a result, the differential mode was normally propagated, since the circuit model consisted of a conventional transmission line with an increased per-section capacitance, whereas the common-mode was rejected due to the resonance behaviour resulting from the presence of the CSRRs. Figs. 3 and 4 depict the corresponding equivalent electrical models for the described topologies and their differential (odd) and common (even) propagation modes. The parameters $L$ and $C$ model the per-section self-inductance and capacitance of the microstrip lines, whereas $C_{C}$ and $L_{C}$ represent the parallel resonant tank corresponding to the CSRRs. The mutual capacitance (crosstalk) is modelled by $C_{m}$, whereas the mutual inductance between transmission lines, $L_{m}$, was ignored for the purpose of simplicity. As for the CSRR-CM-DM topologies, as a parallel notch resonance arises in both propagation modes, a stop-band frequency response is expected. Notice that the expected stop-band resonance frequency is very similar for both propagation modes, since the capacitance $C_{m}$ has a low impact on the other circuit elements. However, the CSRR-CM case presented a dual 
behaviour, since the differential-mode was normally propagated, due to its conventional transmission line model, whereas the common-mode was rejected due to the resonance of the CSRRs.

Figs. 5(a) and (b) show the results of the experimental and electromagnetic simulation (using the commercial Agilent Momentum simulator) with regard to the insertion losses for the differential mode (Sdd21) and common mode $(\operatorname{Scc} 21)$ of the proposed topologies. The EMI stop-band filter frequency response was measured with an Agilent FieldFox N9916A microwave analyser using the vector network analyser mode, according to the method described in [12]. As expected, the CSRR-CM-DM filtered both Sdd21 and Scc21 with measured attenuation on the order of -50 dB. At the same time, the CSRR-CM RFI filter allowed the differential mode and rejected the common mode reaching a stop-band level of over -50 $\mathrm{dB}$.

\section{SIGNAL INTEGRITY PERFORMANCE}

It is well-known that non-homogenous ground planes degrade signal integrity (SI) performance in PCBs. In order to investigate the impact of the CSRRs etched on the ground plane of the differential lines, SI performance was assessed by means of a standard time-domain eye-diagram metric. Specifically, two parameters were assessed as metrics of the eye-pattern quality: maximum eye width (MEW), to evaluate the jitter margin, and maximum eye opening (MEO), to determine the noise margin. Three cases were considered: the homogenous solid ground PCB, the CSRR-CM-DM filter and the CSRR-CM filter.

Several co-simulations of the eye diagram of the proposed PCBs were performed. A $2^{7}$ - 1 non-return-tozero pseudo-random bit sequence (PRBS) was injected at the input port of each differential board under test, whereas the output ports were matched with a $50 \Omega$ load. The transmitted bit-sequence swing and nominal rise/fall time were $600 \mathrm{mV}$ and $0.2 \mathrm{~ns}$, respectively. A PRBS coded at bit rates of $2 \mathrm{~Gb} / \mathrm{s}$ was analysed. Fig. 6 shows the eye diagram plots obtained, and Table 1 reports the SI performance for the boards under test. The reference microstrip differential lines with homogeneous ground had an $\mathrm{MEO}=569 \mathrm{mV}$ and a MEW=498 ps. The signal integrity degradation caused by the CSRR-CM-DM was 
about $4.4 \%$ for the MEO and 6.6\% for the MEW. Degradation was negligible in the case of the CSRR-CM, at $0.1 \%$ for the MEO and $0.2 \%$ for the MEW. Therefore, CSRRs have a low impact on signal integrity in both CSRR-CM-DM and CSRR-CM topologies in the case of conventional microstrips.

\section{RADIATED EMISSION ANALYSIS}

This section will analyse the EMI radiation of the proposed metamaterial implementations. Fig. 7 shows the experimental setup used to study the RFI near- and far-field radiation from the ground plane of the boards under test, similar to that proposed in [4]. However, in the previous study, the authors used a low bandwidth quarter wavelength monopole antenna to capture the radiated energy. In this study, an ultrawideband (UWB) microstrip monopole antenna was designed to sense radiation from the ground plane up to $8 \mathrm{GHz}$, in the near- and far-field regime, depending on the distance, $r$. The antenna's design was based on the topology detailed in [13]. Figs. 8(a) and (b) illustrate the geometry of the antenna, as well as its experimental performance in terms of the return losses (S11) and voltage standing wave ratio (VSWR). The antenna substrate was a commercial FR4 (dielectric constant, $\varepsilon_{r}=4.6$; thickness, $h=1.53 \mathrm{~mm}$ ) with the following dimensions: $W_{a}=34 \mathrm{~mm} ; L_{a}=40 \mathrm{~mm} ; W_{t}=2.8 \mathrm{~mm} ; L_{g}=12.9 \mathrm{~mm} ; R=10.2 \mathrm{~mm}$; and $d_{a}=0.4 \mathrm{~mm}$. The UWB antenna was located over the centre of the ground plane for each board under test. Port 1 of the VNA was connected to a $B A L-0010$ broadband balun $(200 \mathrm{kHz}-10 \mathrm{GHz})$ to ensure that a differential signal was injected into the prototype under test (ports 1 and 2), whereas port 2 of the VNA was connected to the UWB antenna. The board's output ports (3 and 4) were matched with a $50 \Omega$ load. This methodology made it possible to determine the relative measurements of radiation (by means of the $\mathrm{S} 21$ parameter) in order to compare the impact of radiation on the proposed RFI filter topologies.

Fig. 9(a) shows the EMI radiation from the boards under test in the near-field proximity at $r=1 \mathrm{~cm}$; the far-field radiation was tested at $r=10 \mathrm{~cm}$ (see Fig. 9(b)). As expected, the radiation level of the solid ground PCB was lower than that of the CSRR filters. The CSRR-CM-DM had the highest average level of radiation. In particular, the level was higher in the vicinity of the resonance frequency of the CSRRs (2.4 
$\mathrm{GHz}$ ) and their corresponding multiple frequencies. This trend was observed in both field regimes and is due to the higher number of etched rings (factor 2 with regard to the CSRR-CM topology) and the absorption of the conducted differential mode. A maximum difference value of $30 \mathrm{~dB}$ was observed for $r=1 \mathrm{~cm}$, whereas a maximum difference of $38 \mathrm{~dB}$ was observed for $r=10 \mathrm{~cm}$. The UWB antenna ensured the same degree of reception of the radiated signal in order to avoid distortion in the entire frequency range from 3 to $8 \mathrm{GHz}$. Fig. 10 illustrates the simulated far-field maximum radiated electric field, $\mathrm{E}_{\max }$, for the microstrip coupled lines, according to:

$$
E_{\max }=\sqrt{\left(E_{\phi, \max }\right)^{2}+\left(E_{\theta, \max }\right)^{2}}
$$

where $E_{\max }$ is calculated taking into account the maximum electric field components in both $\phi$ and $\theta$ coordinates, $E_{\phi, \max }$, and $E_{\theta, \max }$. The results show a maximum value for the CSRR-CM-DM equal to 99.5

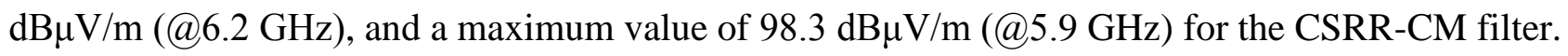

In order to evaluate the behaviour of the different topologies as effective radiators, a far-field analysis of the gain and efficiency of the proposed topologies was performed. As can be seen in Fig. 11, the average gain was similar for all topologies. However, efficiency was significantly impacted by topology. The maximum efficiency for the homogeneous ground plane PCB was $23 \%$, whereas it was $60.4 \%$ for the CSRR-CM-DM and $62.3 \%$ for the CSRR-CM.

\section{CONCLUSION}

Two CSRR RFI filters were studied and compared as differential- and common-mode EMI-mitigation solutions. CSRR-CM-DM and CSRR-CM PCB prototypes were designed, simulated and tested in terms of their frequency response, signal integrity, and near- and far-field radiation impact with regard to a reference (solid ground) board. The results indicate that the CSRR-CM-DM topology had a low impact in terms of 
noise and jitter margin, whereas the impact of the CSRR-CM topology was negligible. Similar radiation levels were observed between both proposed topologies, although the CSRR-CM-DM had the highest average level of radiation.

\section{ACKNOWLEDGEMENTS}

This work was supported by the Spanish Ministry of Economy and Competitiveness (MINECO) under Project TEC2013-41996-R and the Catalan Agency for Administration of University and Research Grants (AGAUR) under Grant 2014 SGR 375. 


\section{REFERENCES}

[1] De Paulis, F., L. Raimondo, S. Connor, B. Archambeault and A. Orlandi, 'Compact Configuration for Common Mode Filter Design based on Planar Electromagnetic Bandgap Structures', IEEE Trans. on Electromagnetic Compatibility, Vol. 54, 646-654, June 2012.

[2] Tsai, C.-H. and T.-L., Wu, 'A Broadband and Miniaturized Common-Mode Filter for Gigahertz Differential Signals Based on Negative-Permittivity Metamaterials', IEEE Trans. on Microwave Theory and Techniques, Vol. 58, 195-202, January 2010.

[3] Falcone, F., T. Lopetegi, J. D. Baena, R Marqués, F. Martín and M. Sorolla, 'Effective negative- $\varepsilon$ stopband microstrip lines based on complementary split ring resonators', IEEE Microwave and Wireless Components Letters, Vol. 14, 280-282, June 2004.

[4] Bait-Suwailam, M. M. and O. M. Ramahi, 'Ultrawideband Mitigation of Simultaneous Switching Noise and EMI Reduction in High-Speed PCBs Using Complementary Split-Ring Resonators', IEEE Trans. on Electromagnetic Compatibility, Vol. 54, 389-396, April 2012.

[5] Zhu, H.-R. and J.-F. Mao, 'Localized Planar EBG Structure of CSRR for Ultrawideband SSN Mitigation and Signal Integrity Improvement in Mixed-Signal Systems', IEEE Trans. on Components, Packaging and Manufacturing Technology, Vol. 3, 2092-2100, December 2013.

[6] Pérez, D., I. Gil, J. Gago, R. Fernández-Garcia, J. Balcells, D. González, N. Berbel and J. Mon, 'Reduction of Electromagnetic Interference Susceptibility in Small-Signal Analog Circuits Using Complementary Split-Ring Resonators', IEEE Trans. on Components, Packaging and Manufacturing Technology, Vol. 2, 240-247, February 2012. 
[7] Gil, I. and R. Fernández-García, 'Electromagnetic interference reduction in printed circuit boards by using metamaterials: a conduction and radiation impact analysis', Journal of Electromagnetic Waves and Applications, Vol. 28, 378-388, February 2014.

[8] Pachon, A. and F. Silva, 'Ethernet 10GBASE-T common mode filter based on metamaterial', EMC Europe 2011 York, 728-731, September 2011.

[9] Gil, I. and R. Fernández-García, 'Differential and common-mode filters based on metamaterials resonators', 2013 International Symposium on Electromagnetic Compatibility (EMC Europe), 10181021, Bruges (Belgium), September 2013.

[10] Marqués, R., F. Mesa, J. Martel and F. Medina, 'Comparative Analysis of Edge- and BroadsideCoupled Split Ring Resonators for Metamaterial Design-Theory and Experiments', IEEE Trans. Antennas Propag., Vol. 51, No. 10, pp. 2572-2581, Oct. 2003.

[11] Baena, J. D., J. Bonache, F. Martín, R. Marqués, F. Falcone, T. Lopetegi, M. A. G. Laso, J. GarcíaGarcía, I. Gil, M. Flores Portillo and M. Sorolla, 'Equivalent-circuit models for split-ring resonators and complementary split-ring resonators coupled to planar transmission lines', IEEE Transactions on Microwave Theory and Techniques, Vol. 53, pp. 1451-1461, April 2005.

[12] Bockelman, D. E. and W. R. Eisenstadt, 'Combined differential and common-mode scattering parameters: theory and simulation', IEEE Trans. Microwave Theory Tech., Vol. 43, 1530-1539, July 1995. 
[13] Liu, J., S. Gong, Y. Xu, X. Zhang, C. Feng and N. Qi, 'Compact printed ultra-wideband monopole antenna with dual band-notched characteristics', Electronics Letters, Vol. 44, 710-711, June 2008. 


\section{FIGURE CAPTIONS}

Fig. 1. Topology of two coupled CSRRs and relevant dimensions. Metallization zones are depicted in grey.

Fig. 2. (a) Differential microstrip coupled lines. This topology is common to all three boards under test. (b) 6-stage CSRR-CM-DM ground plane filter. (c) 6-stage CSRR-CM ground plane filter

Fig. 3. CSRR-CM-DM distributed circuit models for the: (a) unit cell, (b) differential mode and (c) common mode.

Fig. 4. (a) CSRR-CM distributed circuit models for the: (a) unit cell, (b) differential mode and (c) common mode.

Fig. 5. Experimental and electromagnetic simulation results for the insertion losses for the differential mode (Sdd21) and common mode (Scc21) of the (a) CSRR-CM-DM and (b) CSRR-CM filters.

Fig. 6. Eye diagrams generated for a bit rate of $2 \mathrm{~Gb} / \mathrm{s}$. (a) Reference solid ground board, (b) CSRR-CMDM filter case and (c) CSRR-CM filter case.

Fig. 7. Experimental setup used to study EMI radiation from metamaterials and homogeneous ground PCBs.

Fig. 8. (a) Geometry of the proposed UWB monopole antenna. (b) Experimental result of the return losses and VSWR of the proposed radiation test antenna.

Fig. 9. Measured S21 magnitude, according to the radiation measurement setup detailed in Figure 7. The 
microstrip reference, EBG ground and CSRR ground boards were tested at different distance test points: (a) near-field $(\mathrm{r}=1 \mathrm{~cm})$; (b) far-field $(\mathrm{r}=10 \mathrm{~cm})$.

Fig. 10. Simulated far-field maximum radiated electric field for the reference microstrip coupled lines, the CSRR-CM-DM filter and the CSRR-CM filter.

Fig. 11. Antenna gain and efficiency corresponding to (a) reference solid ground board, (b) CSRR-CM-DM filter case and (c) CSRR-CM filter case.

Table I. Comparison of signal integrity performance for the three boards under test 
Table I.

\begin{tabular}{|c|l|l|}
\hline \multirow{2}{*}{ Implementation } & \multicolumn{2}{|c|}{ Bit rate=2 Gb/s } \\
\cline { 2 - 3 } & MEW (ps) & MEO (mV) \\
\hline Reference & 498 & 569 \\
\hline CSRR-CM-DM & $465(6.6 \%)$ & $544(4.4 \%)$ \\
\hline CSRR-CM & $497(0.2 \%)$ & $569(0.1 \%)$ \\
\hline
\end{tabular}

